\title{
Die Systemische Behandlung des metastasierten Nierenzellkarzinoms - Zurück in die Zukunft?
}

\section{Systemic Treatment of Metastatic Renal Cell Cancer - Back to the Future?}

\author{
Autoren \\ Institut

\section{P. Ivanyi, V. Grünwald} \\ Klinik für Hämatologie, Hämostaseologie, Onkologie und Stammzelltransplantation, Medizinische Hochschule Hannover, \\ Hannover
}

Schlüsselwörter
Metastasiertes
Nierenzellkarzinom
Axitinib
Bevacizumab/IFNa
Everolimus
Pazopanib
Sorafenib
Sunitinib
Temsirolimus
Key words
metastatic renal cell
carcinoma
axitinib
bevacizumab/IFNa
everolimus
pazopanib
sorafenib
temsirolimus

Bibliografie

DOI http://dx.doi.org/ 10.1055/s-0041-106136

Online-Publikation: 10.11.2015

Akt Urol 2015; 46: 467-472

(c) Georg Thieme Verlag KG

Stuttgart · New York

ISSN 0001-7868

Korrespondenzadresse

Prof. Dr. med. Viktor Grünwald

Klinik für Hämatologie,

Hämostaseologie,

Onkologie und Stammzelltransplantation

Medizinische Hochschule

Hannover

Carl-Neuberg-Straße 1

30625 Hannover

Tel.: + 49/511/5329196

Fax: + 49/511/532 2501

Gruenwald.Viktor@mh-

hannover.de

\section{Zusammenfassung}

$\nabla$

Zur systemischen Therapie des metastasierten Nierenzellkarzinoms (mNCC) stehen gegenwärtig eine Vielzahl von Therapeutika zur Verfügung. Die Zytokintherapie stellte die erste Generation der medikamentösen Tumortherapie des mNCC dar. Erst mit der Entwicklung der zielgerichteten Therapie in der letzten Dekade konnte eine deutliche Verbesserung der Tumortherapie erzielt werden und hat zur Entwicklung der sequenziellen Therapie des mNCC geführt. Aktuell stehen 7 zielgerichtete Medikamente zur Verfügung (Axitinib, Bevacizumab/IFN $\alpha$, Everolimus, Pazopanib, Sorafenib, Sunitinib und Temsirolimus). Im Zuge der Individualisierung der Therapie stellt die Auswahl des geeigneten Medikamentes die größte Herausforderung für den Behandler dar. Kenntnis der Datenlage, sowie individuelle Faktoren bilden dabei die Grundlage der Medikamentenwahl.

Die Suche nach dem besten Inhibitor oder Kombination hat die letzten Jahre der klinischen Forschung geprägt, ohne dass ein wesentlicher Fortschritt erzielt werden konnte. Stattdessen läutet die aktuelle Entwicklung neuer spezifischer Immuntherapeutika die Entwicklung der nächsten Stufe der Tumortherapie des mNCC ein. Erste Studien werden für dieses Jahr erwartet, weitere Studien zur Optimierung dieser neuen Therapieoption werden folgen.

\section{Einleitung \\ $\nabla$}

Rund $30-40 \%$ der Patienten mit einem Nierenzellkarzinom erleiden (NCC) im Rahmen dieser Erkrankung eine syn-, oder metachrone Metastasierung ( $\mathrm{mNCC}$ ), die einer medikamentösen Therapie bedarf [1]. In der letzten Dekade hat sich die Therapielandschaft des mNCC durch die Entwicklung zielgerichteter Therapien drastisch verändert.

\section{Abstract \\ $\nabla$}

A variety of therapeutic agents are currently available for the systemic treatment of metastatic renal cell carcinoma (mRCC). It was only when targeted treatment was developed in the past decade that a significant improvement was achieved in tumour therapy. This also led to the development of sequential treatment for mRCC.

7 molecular targeted agents are available today (axitinib, bevacizumab/ IFN $\alpha$, everolimus, pazopanib, sorafenib, sunitinib, and temsirolimus). Due to the individualisation of treatment it remains a challenge to choose the most appropriate drug in a given setting, with the choice being based on the knowledge of the relevant clinical data as well as individual patient parameters.

During the recent past, efforts have been made to test different inhibitors or combinations without a major breakthrough. Instead, the development of novel specific immunotherapeutic approaches now heralds the next level of treatment in mRCC. The first significant trial results will be expected this year, and further trials for optimisation of treatment are warranted.
Nachdem mit den Zytokintherapien ein Tumoransprechen von 10-15\% und ein medianes Gesamtüberleben (overall survival [OS]) von rund 13-14 Monaten erzielt werden konnten, wird unter den zielgerichteten Substanzen eine Ansprechrate von ca. 30\% und ein Gesamtüberleben von 30 Monaten erreicht [2-4]. Diese Entwicklung hat faktisch zum Ende der Zytokinära sowohl in der Mono- als auch Kombinationstherapie mit Zytostatika geführt [5]. Allenfalls kann 
eine alleinige Chemotherapie beim sarkomatoid differenzierten mNCC als akzeptable Alternative angesehen werden [6].

Die neue Potenz der gezielten Therapien beim mNCC verbesserte das OS im indirekten Vergleich zur Zytokinära signifikant. Eine populationsbasierte Analyse verglich Zeiträume mit jeweiliger Dominanz von Zytokin,- oder zielgerichteter Therapie und zeigte ein besseres Überleben im Zeitraum der zielgerichteten Therapien (1992-2004: 13 Monaten vs. 2005-2009: 16 Monaten; $\mathrm{p}<0.0001$ ), ähnliche Ergebnisse fanden 2 weitere größere Kohortenanalysen [7-9]. Insbesondere gilt dieser Trend für das klarzellige mNCC und jüngere Patienten $[9,10]$. Da die Mehrzahl der Studien beim klarzelligen NCC durchgeführt wurde, finden sich die drastischen Therapiefortschritte überwiegend bei diesem dominanten histologischen Subtyp.

Aktuell kann mit zielgerichteter Therapie beim mNCC ein Gesamtüberleben von rund 30 Monaten erzielt werden. Ca.10\% der Patienten erzielen eine Langzeitremission, was mit einem medianen OS von 55 Monaten assoziiert ist [11-13]. Insgesamt scheint eine weitere Verbesserung der Prognose von mNCC Patienten nur durch neue Therapieansätze, wie z.B. die Checkpoint-Inhibitoren, erreichbar zu sein.

Die Vielfalt der systemischen Therapieoptionen beim mNCC erfordert bereits heute ein differenziertes Vorgehen. Die Auswahl des „einen“ optimalen Präparates und der „einen“ optimalen Therapiesequenz lässt nicht belegen, hingegen scheint die Auswahl des für den Patienten besten Präparats in der für den Patienten bestmöglichen Sequenz von übergeordneter Bedeutung. Bereits vor Behandlungsbeginn ist z.B. die Risikoklassifizierung eines mNCC Patienten nach dem IMDC-Risiko-Score als Standard

Tab. 1 Prognose Kriterien zur Bestimmung der Risikogruppen (nach Heng et al.) mit der entsprechenden Prognose der jeweiligen Risikogruppe.

\begin{tabular}{|c|c|c|}
\hline \multicolumn{3}{|l|}{ IMDC Kriterien } \\
\hline \multicolumn{3}{|l|}{ Performance Status $<80 \%$} \\
\hline \multicolumn{3}{|c|}{ Intervall von Diagnose bis zur Systemtherapie $<1$ Jahr } \\
\hline \multicolumn{3}{|c|}{ Hämoblobin unterhalb des Normwertes } \\
\hline \multicolumn{3}{|c|}{ Hyperkalziämie } \\
\hline \multicolumn{3}{|c|}{ Neutrophile oberhalb des Normwertes } \\
\hline \multicolumn{3}{|c|}{ Thrombozyten oberhalb des Normwertes } \\
\hline \multicolumn{3}{|c|}{ Prognose nach IMDC Risikogruppen } \\
\hline Anzahl der Risikofaktoren & Risikoprofil & Medianes OS \\
\hline 0 & Gutes Risikoprofil & 43,2 Monate \\
\hline $1-2$ & Intermediäres Risikoprofil & 22,2 Monate \\
\hline $3-6$ & Ungünstiges Risikoprofil & 7,8 Monate \\
\hline
\end{tabular}

einzufordern, der im klinischen Alltag Grundlage für Therapieentscheidungen darstellt ( $\bullet$ Tab. 1) [14]. Anhand der Risikopunkte werden die Patienten in ein gutes, intermediäres oder ungünstiges Risikoprofil eingeteilt. Der IMDC Score ist ebenso in der zweiten Therapielinie etabliert [15].

\section{Erstlinientherapie}

4 Therapeutika sind in Europa beim therapienaiven Patienten, neben IFN $\alpha$ zugelassen: Sunitinib, ein oraler Multi-TyrosinkinaseInhibitor (MTKI), der den vaskulären endothelialen Wachstumsfaktor Rezeptor (VEGFR) 2 und 3, den platelet-derived-growthfactor-receptor (PDGFR) $\alpha$, c-KIT, FLT (Fms-like tyrosine kinase)-3, inhibiert; Pazopanib, ebenfalls ein oraler MTKI, der VEGFR 1-3, c-KIT, PDGFR $\alpha$ und $\beta$ inhibiert; Temsirolimus, ein intravenöser mTOR (mammalian target of rapamycin) Inhibitor, und die Kombination aus Bevacizumab/INF $\alpha$, einem monoklonalen Antikörper gegen den löslichen VEGF in Kombination mit einer unspezifischen Immuntherapie [16-22].

Sunitinib, Pazopanib und Bevacizumab/IFN $\alpha$, sind jeweils für alle Risikogruppen zugelassen. Die $\operatorname{VEGF(R)~Inhibitoren~zeigten~}$ in den Zulassungsstudien Gesamtansprechraten (ORR: komplette Remissionen [CR]+partielle Remissionen [PR]) von ca. 25-30\%, sowie einen klinischen Nutzen (CBR: $\mathrm{CR}+\mathrm{PR}+$ stabile Erkrankungen (SD)) bei etwa 70-79\% der Patienten ( $\odot$ Tab. 2). Der Anteil der kompletten Remissionen lag in den Studien bei $<1 \%$. Das jeweilige PFS lag zw. 8,5-11 Monaten, mit einem Trend zu einem verbesserten OS, wenngleich nachfolgend eingesetzte Therapien ähnlich wirksamer Substanzen wahrscheinlich das Erreichen eines signifikanten OS Vorteils verhinderten [16-19,21,23]. Der einzige mTOR Inhibitor in der Erstliniensituation, Temsirolimus, wurde in einer 3-armigen Phase-3 Studie ausschließlich bei Patienten mit ungünstigem Risikoprofil getestet. Temsirolimus zeigte hier ein PFS von 5,5 Monaten mit signifikanter Verbesserung des OS (10,9 Monate) und wurde entsprechend als einzige Substanz ausschließlich für Patienten mit ungünstigem Risikoprofil in Erstliniensituation zugelassen [20] ( $\bullet$ Tab. 2).

Aufgrund von fehlenden vergleichenden Studien kann zum gegenwärtigen Zeitpunkt im Hinblick auf das Tumoransprechen keine überlegene Erstlinientherapie definiert werden ( $\bullet$ Tab. 2 u. 4). Eine direkt vergleichende Phase-3-Studie erfolgte lediglich zwischen Pazopanib und Sunitinib (COMPARZ Studie), in der zwar Unterschiede bei der Verträglichkeit zugunsten von Pazopanib gezeigt werden konnte, nicht jedoch bei der Wirksamkeit $[12,23]$.

Tab. 2 Gegenüberstellende Übersicht über die Effizienz und Sicherheit von Therapeutika mit Zulassung in der Erstliniensituation beim mNCC. Gezeigt sind die jeweiligen Verum-Arme der Phase-3 Prüfung.

$\begin{array}{llllllll} & \begin{array}{l}\text { Bev/IFN } \\ \text { (AVOREN) }\end{array} & \begin{array}{l}\text { BEV/IFN } \\ \text { (CALGB) }\end{array} & \begin{array}{l}\text { Pazopanib } \\ \text { (VEGF 105192) }\end{array} & \begin{array}{l}\text { Pazopanib } \\ \text { (COMPARZ) }\end{array} & \begin{array}{l}\text { Sunitinib } \\ \text { (COMPARZ) }\end{array} & \begin{array}{l}\text { Sunitinib } \\ \text { (NCT00098657) }\end{array} & \begin{array}{l}\text { Temsirolimus } \\ \text { (ARCC) }\end{array} \\ \text { Ungünstiges Risiko [20] }\end{array}$


Unter Beachtung des IMDC Risikos stellt Temsirolimus zwar die einzige Substanz dar, die in der Erstlinie bei ungünstigem Risiko das OS nachweislich verbesserte, jedoch erscheint Sunitinib in gleicher Konstellation als nahezu gleichwertige Therapiealternative. Das erweiterte Zulassungsprogramm für Sunitinib umfasst 3225 Patienten, darunter 373 Patienten mit ungünstigem Risikoprofil, deren PFS mit 4,1 Monaten eine ähnliche Wirksamkeit wie unter Temsirolimus erreichte [20,24,25]. Aufgrund der COMPARZ Studie kann auch unter Vorbehalt auf eine dem Sunitinib ähnliche Sicherheit und Effektivität von Pazopanib in dieser Situation zurückgeschlossen werden [12,23]. Zu Bevacizumab/IFN $\alpha$ in Erstliniensituation bei ungünstigem Risikoprofil stehen gegenwärtig nicht ausreichend Daten zum rechtfertigen Einsatz dieser Kombination zur Verfügung ( $\bullet$ Tab. 3).

\section{Zweitlinientherapie}

Bei vorbehandelten Patienten, respektive bei Patienten in der zweiten Therapielinie, sind 4 Substanzen zugelassen und entsprechend getestet: der MTKI Axitinib, ein Inhibitor von VEGFR

Tab. 3 Übersicht zur Sequenztherapie bzw. Systemtherapieoptionen beim mNCC gemäß Risikoprofil und Therapielinie (in Anlehnung an die ESMO Therapieempfehlungen [50]).

\begin{tabular}{|c|c|c|c|}
\hline Therapielinie & Risikoprofil & Standard & Option \\
\hline \multicolumn{4}{|l|}{ Erstlinie } \\
\hline & Gut/intermediär & $\begin{array}{l}\text { Bevacizumab/IFN } \alpha \\
\text { Pazopanib } \\
\text { Sunitinib }\end{array}$ & HD-IL2 \\
\hline & Ungünstig & Temsirolimus & $\begin{array}{l}\text { Sunitinib } \\
\text { Pazopanib } \\
\text { Sorafenib }\end{array}$ \\
\hline \multicolumn{4}{|c|}{ Zweitlinientherapie } \\
\hline & Nach Zytokinen & $\begin{array}{l}\text { Axitinib } \\
\text { Pazopanib } \\
\text { Sorafenib }\end{array}$ & \\
\hline & Nach VEGF-Versagen & $\begin{array}{l}\text { Everolimus } \\
\text { Axitinib }\end{array}$ & \\
\hline & Nach mTOR Inhibition * & $\begin{array}{l}\text { Axitinib } \\
\text { Pazopanib } \\
\text { Sorafenib } \\
\text { Sunitinib }\end{array}$ & \\
\hline \multicolumn{4}{|c|}{ Drittlinientherapie } \\
\hline & $\begin{array}{l}\text { Nach 2-maligem VEGF } \\
\text { Versagen }\end{array}$ & $\begin{array}{l}\text { Everolimus } \\
\text { MTKI* }^{*}\end{array}$ & \\
\hline & $\begin{array}{l}\text { Nach VEGF und mTOR } \\
\text { Versagen }\end{array}$ & Sorafenib & $\begin{array}{l}\text { Re-Chellan- } \\
\text { ge mit MTKI }\end{array}$ \\
\hline
\end{tabular}

1-3 der zweiten Generation, zugelassen nach Therapieversagen von Sunitinib oder Zytokinen; der orale MTKI Sorafenib, der die RAS/RAF-Kinasen, VEGFR 2-3, PDGFR und c-KIT inhibiert und nach Zytokinversagen zugelassen ist. Pazopanib nach Zytokinversagen, sowie der orale mTOR Inhibitor Everolimus, zugelassen nach Progress einer anti-VEGF(R)-Therapie $[26,27]$. Die Zulassungsstudien zeigten ein PFS im Bereich von 4,7-6,7 Monaten, wobei durch den hohen Anteil von wirksamen Folgetherapien das Signifikanzniveau für den sekundären Endpunkt des OS nicht erreicht werden konnte [26-28] (๑ Tab. 4).

Da heutzutage eine alleinige Zytokintherapie in der Erstlinie kaum mehr erfolgt, ist die Vorbehandlung mit einem $\operatorname{VEGF(R)}$ Inhibitor von wesentlicher Bedeutung und stellt somit die Ergebnisse zweier Zulassungsstudien, AXIS und RECORD-1, in den Vordergrund. Die AXIS Studie zeigte die Wirksamkeit von Axitinib im Vergleich zu Sorafenib in der reinen zweiten Therapielinie [26]. Das PFS von Axitinib zeigte sich hier überlegen (PFS: Axitinib: 6,7 Monate vs. Sorafenib: 4,7 Monate; $p<0,0001$ ), bei nahezu gleichem OS (Axitinib: 20,1 Monate vs. Sorafenib: 19,2 Monaten, $\mathrm{p}=0,3744)[26]$. Auch zeigte Axitinib im direkten Vergleich zu Sorafenib geringere nebenwirkungsassoziierte Therapieabbruch(3,9 vs. $8,2 \%$ ), und Dosisreduktionsraten ( 31 vs. $52 \%$ ).

Everolimus wurde in der RECORD-1 Studie in einem gemischten multipel vorbehandeltem Kollektiv getestet. Das PFS betrug 4,9 Monate und weist damit eine ähnliche Marge wie die anderen Therapieoptionen auf. Insgesamt muss die Wirksamkeit der Zweitlinie als moderat bewertet werden. Ein Vergleich zwischen Everolimus und MTKI in der Zweitlinie ist bisher nicht erfolgt. Allerdings erfolgte ein direkter Vergleich in der 2. Linientherapiesituation für den mTOR Inhibitor Temsirolimus zum MTKI Sorafenib (INTROSECT Studie), der keinen signifikanten Vorteil für das PFS von Temsirolimus zeigen konnte (4,3 vs. 3,9 Monate, $p=0,19$ ). Der sekundäre Endpunkt OS zeigte hier jedoch einen Vorteil zugunsten von Sorafenib (OS: 12,3 vs. 16,6 Monate, p=0,01), was gegenwärtig nicht sicher beurteilt werden kann [29].

Zusammenfassend sind somit im Wesentlichen Axitinib als auch Everolimus die Therapeutika in der zweiten Therapielinie. Je nach Erstlinientherapie können Pazopanib und Sorafenib Behandlungsalternativen darstellen. Die Auswahl der jeweiligen Therapieoption erfolgt individuell und richtet sich sowohl nach der zu erwartenden Effektivität als auch der bisherigen Verträglichkeit der Therapien ( $\bullet$ Tab. 3 ).

Drittlinientherapie und weitere Linien der Behandlung Zur gezielten Therapie in der dritten Therapielinie zeigte die GOLD-Studie (Phase-3) klare Rationalen [30]. Bei Patienten mit

\begin{tabular}{|c|c|c|c|c|}
\hline & $\begin{array}{l}\text { Axitinib } \\
\text { (AXIS) nach MTKI oder } \\
\text { Zytokinen }[26,49]\end{array}$ & $\begin{array}{l}\text { Everolimus } \\
\text { (RECORD1) } \\
\text { nach MTKI [27] }\end{array}$ & $\begin{array}{l}\text { Sorafenib } \\
\text { (TARGET) } \\
\text { nach Zytokine n }[22,28]\end{array}$ & $\begin{array}{l}\text { Pazopanib } \\
\text { (VEG105192) nach } \\
\text { Zytokinen }[17,48]\end{array}$ \\
\hline $\begin{array}{l}\text { Ansprechraten, \% } \\
(\mathrm{ORR}=\mathrm{CR}+\mathrm{PR})\end{array}$ & 19 & 1 & 10 & 39 \\
\hline $\begin{array}{l}\text { Clinical Benefit Rate, \% } \\
(C R+P R+S D)\end{array}$ & 46 & 64 & 84 & NA \\
\hline PFS, Mo. & 6,7 & 4,0 & 5,5 & 7,4 \\
\hline OS, Mo. & 20,1 & 14,8 & 19,3 & 22,7 \\
\hline $\begin{array}{l}\text { Abbruchraten } \\
\text { (AE assoziiert),\% }\end{array}$ & 4 & 10 & 10 & 19 \\
\hline $\begin{array}{l}\text { Dosisreduktionsraten } \\
\text { (AE assoziiert)\% }\end{array}$ & 31 & 7 & 13 & NA \\
\hline Nebenwirkungen CTC Grad 3/4,\% & NA & NA & NA & 33 \\
\hline
\end{tabular}

Tab. 4 Übersicht über die Effizienz und Sicherheit von Therapeutika mit Zulassung in der 2. Liniensituation. Gezeigt sind die jeweiligen Verum-Arme der Phase-3 Prüfung. 
mindestens 2 Vortherapien (eine MTKI und eine MTOR Inhibitor Therapie) zeigte der experimentelle VEGFR und fibroblastgrowth-factor receptor (FGFR) Inhibitor Dovitinib im Vergleich zu Sorafenib ein ähnliches PFS von 3,7 vs. 3,6 Monaten $(p=0,36)$, woraufhin die klinische Weiterentwicklung von Dovitinib eingestellt wurde [30]. Im Umkehrschluss müssen diese Ergebnisse als klares Signal für die Wirksamkeit der VEGFR-Inhibition in der dritten Therapielinie gewertet werden, zumindest nach der Vorsequenz MTKI-mTOR Inhibitor. Weitere Mosaike für die Wirksamkeit von VEGFR Inhibition in der dritten Therapielinie lassen sich auch von präliminären Daten von Pazopanib in der Drittlinie ableiten ( $n=28$, PFS: 16 Monate) [31].

Da die Zweitlinientherapie entweder aus einem Wechsel der Substanzklasse (mTOR Inhibitor) oder aus weiterem Einsatz eines MTKI bestehen kann, stehen für die Drittlinientherapie somit Therapien mit unterschiedlicher Evidenz zur Verfügung. Während die höchste Evidenz für Sorafenib auszumachen ist, zeigt eine Subgruppe der RECORD-1 Studie (ca. 20\% der Patienten) eine Rationale für den mTOR Inhibitor Everolimus nach dem Versagen zweier vorheriger MTKI [27]. Ein direkter Vergleich zwischen Sorafenib und Everolimus in der Drittlinie steht allerdings nicht zur Verfügung.

Zusammenfassend ist eine Drittlinientherapie als sinnvoll zu erachten, was auch eine Analyse des ,international mRCC database consortium“ mit 2705 Patienten zeigte, in der sich 16\% der Patienten in Drittlinientherapiesituation befanden, mit einem PFS von 4,4 Monaten und ein OS von 18 Monaten [32]. Insbesondere Patienten mit niedrigem Risikoprofil scheinen von einer Drittlinientherapie zu profitieren [33].

Im Hinblick auf die 4. Therapielinie lässt sich vermuten, dass auch hier eine gewisse Aktivität zu erwarten ist. Legt man der Gesamtbeurteilung den Vergleich mit Placebo-behandelten Studien zugrunde, so schneiden im studienübergreifenden Vergleich Patienten mit einer aktiven Behandlung deutlich besser ab. Aus der RECORD-1 Studie wird unter Placebo ein PFS von 1,9 Monaten erzielt, was im Vergleich zum Drittlinien PFS der GOLD Studie von 3,6-3,7 Monaten unterdurchschnittlich ist [27,30]. Insofern sollte das Ziel die Dauertherapie der Patienten sein.

\section{Die optimale Sequenz, oder die für den Patienten} optimale Therapie?

Auch wenn in den vergangenen Jahren viel über die optimale Therapiesequenz, über Vor-, und Nachteile eines Wechsels der Therapiemodalitäten oder der Inhibitionsspektren diskutiert wurde, kann zum gegenwärtigen Zeitpunkt anhand der Datenlagen nicht die optimale Therapiesequenz apriori definiert werden [34]. Stattdessen sollte in jeder Linie die für die jeweilige Situation beste Therapieentscheidung getroffen werden.

Während in der Erstlinie die Mehrzahl der Patienten einen MTKI erhält ging die RECORD-3 Studie der Frage zum Einsatz von Sunitinib oder Everolimus in der Erstlinie nach. In dieser Situation ist Everolimus dem Sunitinib mit einem PFS von 7,9 Monaten vs. 10,7 Monaten klar unterlegen. Auch das vorläufige OS zeigte einen negativen Trend (22 vs. 32 Monate) [35]. Dies unterstreicht die Überlegenheit der VEGFR Inhibition gegenüber der mTOR Inhibition in der Erstlinie, entsprechend sollte Everolimus hier nicht zum Einsatz kommen [35].

Da in der Zweitlinie sowohl MTKI als auch Everolimus eingesetzt werden können, gibt es keine definierte Sequenz [34,36]. Allerdings erscheinen primäre Bedenken gegenüber Kreuzresistenzen bei einer Abfolge MTKI-MTKI als unbegründet, was sich aus der SWITCH Studie ableiten lässt [37]. Sowohl die Sequenz Suni-
tinib-Sorafenib als auch die Sequenz Sorafenib-Sunitinib konnte eine Wirksamkeit für die MTKI-MTKI Sequenz belegen, wenngleich auch hier keine optimale Sequenz identifiziert werden konnte [37]. Zusammenfassend sind entsprechend der ESMO Therapieleitlinien die Möglichen Therapiesequenzen in $\bullet$ Tab. 3 dargestellt.

Zulassungsstudien, populationsbasierte-, und Meta-Analysen zeigen, dass die Sequenztherapie beim mNCC ein etablierter Standard ist und suggerieren das möglicherweise neben der Tumorbiologie die prinzipielle Exposition zu möglichst vielen aktiven Substanzen für ein bestmögliches Therapieansprechen mitentscheidend sein könnte $[30,32,38]$. Eine globale populationsbasierte deskriptive Analyse mit 2106 Patienten zeigte, das gerade einmal 43\% der Patienten eine zweite Therapiesequenz erhielten, was sich nicht ausschließlich durch tumorbedingte Mortalität in und um die Erstliniensituation erklärt [36]. Ähnliche Ergebnisse zeigte auch ein deutsches Patientenregister. Hieraus lässt sich ableiteten, dass neben einem effektivem Therapeutikum mutmaßlich auch der Patientenadherence, der supportiven Therapie, der Patientenkomorbidität, als auch der Antizipation von Toxizitäten ein besonderer Stellenwert in der mNCC Therapie einzuräumen ist [39]. Dies wird umso deutlicher bei Betrachtung der Dosisreduktionsraten von 7-50\% bzw. der Therapieabbruchraten von 3-28\% die die gezielten Therapien in den Zulassungsstudien zeigten $[16,18,20,26,27,30]$. Wenn- gleich die MTKI einfach ambulant zu applizierende Substanzen sind besitzen diese ein erhebliches Nebenwirkungspotenzial mit auch erhöhtem Mortalitätspotenzial [40]. Insofern ist auch das individuelle Komorbiditätsprofil ein entscheidender Faktor bei der Therapieauswahl [39].

Eine retrospektive Analyse einer offenen Kohortenstudie mit 291 Patienten generierte erste Signale, das nahe liegender Weise Nebenwirkungen überproportional mit Dosis-reduktionen und darüber hinaus mit einem reduziertem OS in Zusammenhang stehen könnten [41]. Nebenwirkungsmanagement und die daraus resultierende Patientenzufriedenheit sind somit essentiell und werden immer wichtiger Parameter. Die COMPARZ Studie zeigte bereits Signale im Hinblick auf deutlich unterschiedliche Therapieverträglichkeiten zweier ähnlicher MTKI, Sunitinib und Pazopanib [23]. Unterstützt werden diese Ergebnisse durch die PISCES-Studie, die eine Präferenz der Patienten zur Einnahme von Pazopanib im Vergleich zu Sunitinib zeigte [42].

Kenntnis des Toxizitätsprofils, des Komorbiditätsprofils und Erfahrungen im Nebenwirkungsmanagement sind somit wichtige Voraussetzung zum Erreichen optimaler Therapieergebnisse, sodass die Wahl des richtigen Therapeutikums für den Patienten in der entsprechenden Situation zumindest genau so relevant erscheint wie die offene Überlegung um die bestmögliche Therapiesequenz.

\section{Ausblick - Zurück in die Zukunft?}

$\nabla$

Nachdem die letzte Dekade neue Therapieoptionen für das mNCC hervorbrachte, bleibt die Therapie des mNCC weiterhin ein dynamisches und hochinnovatives Therapiefeld. Nach einer Suche nach der besten Substanz, Kombinationen gezielter Therapien oder Sequenz beim mNCC, hat das mediane Gesamtüberleben mit 29-30 Monate in aktuellen Studien ein Plateau erreicht [11-13]. Eine weitere Verbesserung des Ergebnisses erscheint in dieser Situation nur durch den Einsatz neuer Substanzen mit neuen Mechanismen denkbar [43]. 
Neben neuen molekularen Therapieansätzen befinden sich bereits neue gezielte Therapien erfolgreich in der frühen klinischen Prüfung. Cabozantinib ist ein Inhibitor von c-MET und VEGFR, der bei stark vorbehandelten Patienten mit mNCC ein PFS von 12,9 Monaten und ein OS von 15 Monaten erzielte und dessen Phase-3 Daten in der Zweit- bis Drittlinie in diesem Jahr erwartet werden [44].

Die wohl größte und spannendste Entwicklung der letzten Jahre ist allerdings der Einsatz von neuen spezifischen Immuntherapeutika, den sogenannten Checkpoint Inhibitoren. Im Gegensatz zur unspezifischen Zytokin-Immunmodulation der 80er Jahre, können Checkpoint Inhibitoren die tumorbedingte Immunblockade aufheben und damit Langzeit-remissionen induzieren. Der Einsatz der Checkpoint-Inhibitoren hat bereits die therapeutische Landschaft des malignen Melanoms nachhaltig verändert. $\mathrm{Zu}$ den Checkpoint-Inhibitoren, die sich gegenwärtig in klinischer Prüfung beim mNCC befinden, gehören vor allem Antikörper gegen PD-1 und PD-1L, sowie CTLA-4. Teils erfolgt bereits die Prüfung von Kombinationen dieser Antikörper. Bereits in Phase-1 Studien zeigte sich ein erstaunliches Tumoransprechen in verschiedenen Tumoren. Nivolumab (Anti-PD1) zeigte bei vortherapierten mNCC Patienten bei $27 \%$ der Patienten ein Tumoransprechen von über 24 Wochen [45]. In einer Analyse einer Expansionskohorte einer Phase-1 Studie mit Nivolumab zeigten schwer vortherapierte mNCC Patienten ein erstaunliches OS von 22,4 Monaten [46].

Zusammenfassend scheint somit zukünftig neben der Identifizierung neuer molekularer Ziele wie z.B. c-MET, der spezifischen Immuntherapie möglicherweise ein hoher Stellenwert zuzukommen, nachdem die unspezifische Immuntherapie diesen bei der Behandlung des mNCC nahezu vollständig verloren hat.

Interessenkonflikt: PI: Beratertätigkeit: Bayer und Novartis. Honorarverträge: Bayer, Novartis, GSK. VG: Honorarverträge: Novartis, Pfizer, GSK, BMS. Beratertätigkeit: Novartis, Pfizer, GSK, BMS, MSD, Bayer.

\section{Literatur}

1 Lam JS, Shvarts O, Leppert JT et al. Renal cell carcinoma 2005: new frontiers in staging, prognostication and targeted molecular therapy. J Urol 2005; 173: 1853-1862

2 Yang JC, Sherry RM, Steinberg SM et al. Randomized study of high-dose and low-dose interleukin-2 in patients with metastatic renal cancer. J Clin Oncol 2003; 21: 3127-3132

3 Minasian LM, Motzer RJ, Gluck L et al. Interferon alfa-2a in advanced renal cell carcinoma: treatment results and survival in 159 patients with long-term follow-up. J Clin Oncol 1993; 11: 1368-1375

4 Coppin C, Porzsolt F, Awa A et al. Immunotherapy for advanced renal cell cancer. Cochrane Database Syst Rev 2005 CD001425

5 Gore ME, Griffin $C L$, Hancock B et al. Interferon alfa-2a versus combination therapy with interferon alfa-2a, interleukin-2, and fluorouracil in patients with untreated metastatic renal cell carcinoma (MRC RE04/ EORTC GU 30012): an open-label randomised trial. Lancet 2010; 375: 641-648

6 Buti S, Bersanelli M, Sikokis A et al. Chemotherapy in metastatic renal cell carcinoma today? A systematic review. Anticancer Drugs 2013; 24: $535-554$

7 Wahlgren T, Harmenberg $U$, Sandstrom $P$ et al. Treatment and overall survival in renal cell carcinoma: a Swedish population-based study (2000-2008). Br J Cancer 2013; 108: 1541-1549

8 Soerensen AV, Donskov F, Hermann GG et al. Improved overall survival after implementation of targeted therapy for patients with metastatic renal cell carcinoma: results from the Danish Renal Cancer Group (DARENCA) study-2. Eur J Cancer 2014; 50: 553-562

9 Pal SK, Nelson RA, Vogelzang $N$. Disease-specific survival in de novo metastatic renal cell carcinoma in the cytokine and targeted therapy era. PLoS One 2013; 8: e63341
10 Nelson RA, Vogelzang N, Pal SK. A gap in disease-specific survival between younger and older adults with de novo metastatic renal cell carcinoma: results of a SEER database analysis. Clin Genitourin Cancer 2013; 11: 303-310

11 Michel MS, Vervenne $W$, de Santis $M$ et al. SWITCH: A randomized sequential open-label study to evaluate efficacy and safety of sorafenib (SO)/sunitinib (SU) versus SU/SO in the treatment of metastatic renal cell cancer (mRCC). J Clin Oncol 2014; 32: Supplement 4, Abstract 393

12 Motzer RJ, Hutson TE, McCann L et al. Overall survival in renal-cell carcinoma with pazopanib versus sunitinib. N Engl J Med 2014; 370: 1769-1770

13 Grunwald V, McKay RR, Krajewski KM et al. Depth of remission is a prognostic factor for survival in patients with metastatic renal cell carcinoma. Eur Urol 2015; 67: 952-958

14 Heng DY, Xie W, Regan MM et al. Prognostic factors for overall survival in patients with metastatic renal cell carcinoma treated with vascular endothelial growth factor-targeted agents: results from a large, multicenter study. J Clin Oncol 2009; 27: 5794-5799

15 Ko JJ, Xie W, Kroeger $N$ et al. The International Metastatic Renal Cell Carcinoma Database Consortium model as a prognostic tool in patients with metastatic renal cell carcinoma previously treated with first-line targeted therapy: a population-based study. Lancet Oncol 2015; 16: 293-300

16 Motzer RJ, Hutson TE, Tomczak P et al. Sunitinib versus interferon alfa in metastatic renal-cell carcinoma. N Engl J Med 2007; 356: 115-124

17 Sternberg CN, Davis ID, Mardiak J et al. Pazopanib in locally advanced or metastatic renal cell carcinoma: results of a randomized phase III trial. J Clin Oncol 2010; 28: 1061-1068

18 Rini BI, Halabi S, Rosenberg JE et al. Phase III trial of bevacizumab plus interferon alfa versus interferon alfa monotherapy in patients with metastatic renal cell carcinoma: final results of CALGB 90206. J Clin Oncol 2010; 28: 2137-2143

19 Escudier B, Bellmunt J, Negrier $S$ et al. Phase III trial of bevacizumab plus interferon alfa-2a in patients with metastatic renal cell carcinoma (AVOREN): final analysis of overall survival. J Clin Oncol 2010; 28: 2144-2150

20 Hudes G, Carducci M, Tomczak P et al. Temsirolimus, interferon alfa, or both for advanced renal-cell carcinoma. N Engl J Med 2007; 356: 2271-2281

21 Escudier B, Pluzanska A, Koralewski P et al. Bevacizumab plus interferon alfa-2a for treatment of metastatic renal cell carcinoma: a randomised, double-blind phase III trial. Lancet 2007; 370: 2103-2111

22 Escudier B, Eisen T, Stadler WM et al. Sorafenib in advanced clear-cell renal-cell carcinoma. N Engl J Med 2007; 356: 125-134

23 Motzer RJ, Hutson TE, Cella D et al. Pazopanib versus sunitinib in metastatic renal-cell carcinoma. N Engl J Med 2013; 369: 722-731

24 Motzer RJ, Hutson TE, Tomczak P et al. Overall survival and updated results for sunitinib compared with interferon alfa in patients with metastatic renal cell carcinoma. J Clin Oncol 2009; 27: 3584-3590

25 Gore ME, Szczylik C, Porta C et al. Safety and efficacy of sunitinib for metastatic renal-cell carcinoma: an expanded-access trial. Lancet Oncol 2009; 10: 757-763

26 Rini BI, Escudier B, Tomczak P et al. Comparative effectiveness of axitinib versus sorafenib in advanced renal cell carcinoma (AXIS): a randomised phase 3 trial. Lancet 2011; 378: 1931-1939

27 Motzer RJ, Escudier B, Oudard S et al. Efficacy of everolimus in advanced renal cell carcinoma: a double-blind, randomised, placebo-controlled phase III trial. Lancet 2008; 372: 449-456

28 Escudier B, Eisen T, Stadler WM et al. Sorafenib for treatment of renal cell carcinoma: Final efficacy and safety results of the phase III treatment approaches in renal cancer global evaluation trial. J Clin Oncol 2009; 27: 3312-3318

29 Hutson TE, Escudier B, Esteban E et al. Randomized phase III trial of temsirolimus versus sorafenib as second-line therapy after sunitinib in patients with metastatic renal cell carcinoma. J Clin Oncol 2014; 32: 760-767

30 Motzer RJ, Porta C, Vogelzang NJ et al. Dovitinib versus sorafenib for third-line targeted treatment of patients with metastatic renal cell carcinoma: an open-label, randomised phase 3 trial. Lancet Oncol 2014; 15: 286-296

31 Pal SK, Hossain DM, Zhang Q et al. Pazopanib as third line therapy for metastatic renal cell carcinoma: clinical efficacy and temporal analysis of cytokine profile. J Urol 2015; 193: 1114-1121

32 Ko JJ, Choueiri TK, Rini BI et al. First-, second-, third-line therapy for mRCC: benchmarks for trial design from the IMDC. Br J Cancer 2014; 110: $1917-1922$ 
33 Iacovelli R, Farcomeni A, Sternberg CN et al. Prognostic Factors in Patients Receiving Third Line Targeted Therapy for Metastatic Renal Cell Carcinoma. J Urol 2014

34 Escudier B, Eisen T, Porta $C$ et al. Renal cell carcinoma: ESMO Clinical Practice Guidelines for diagnosis, treatment and follow-up. Ann Oncol 2012; 23: (Suppl 7): vii65-vii71

35 Motzer RJ, Barrios CH, Kim TM et al. Phase II randomized trial comparing sequential first-line everolimus and second-line sunitinib versus first-line sunitinib and second-line everolimus in patients with metastatic renal cell carcinoma. J Clin Oncol 2014; 32: 2765-2772

36 Alimohamed N, Lee JL, Srinivas S et al. A population-based overview of sequences of targeted therapy in metastatic renal cell carcinoma. Clin Genitourin Cancer 2014; 12: e127-e131

37 Eichelberg C, Vervenne WL, De Santis $M$ et al. SWITCH: A Randomised, Sequential, Open-label Study to Evaluate the Efficacy and Safety of Sorafenib-sunitinib Versus Sunitinib-sorafenib in the Treatment of Metastatic Renal Cell Cancer. Eur Urol 2015

38 Coppin C, Kollmannsberger C, Le L et al. Targeted therapy for advanced renal cell cancer (RCC): a Cochrane systematic review of published randomised trials. BJU Int 2011; 108: 1556-1563

39 Schmidinger M, Zielinski CC. Novel agents for renal cell carcinoma require novel selection paradigms to optimise first-line therapy. Cancer Treat Rev 2009; 35: 289-296

40 Schutz FA, Je Y, Richards CJ et al. Meta-analysis of randomized controlled trials for the incidence and risk of treatment-related mortality in patients with cancer treated with vascular endothelial growth factor tyrosine kinase inhibitors. J Clin Oncol 2012; 30: 871-877

41 Porta C, Levy A, Hawkins $R$ et al. Impact of adverse events, treatment modifications, and dose intensity on survival among patients with advanced renal cell carcinoma treated with first-line sunitinib: a medical chart review across ten centers in five European countries. Cancer Med 2014; 3: 1517-1526
42 Escudier B, Porta C, Bono P et al. Randomized, controlled, double-blind, cross-over trial assessing treatment preference for pazopanib versus sunitinib in patients with metastatic renal cell carcinoma: PISCES Study. J Clin Oncol 2014; 32: 1412-1418

43 Grunwald V, Merseburger AS. The progression free survival-plateau with vascular endothelial growth factor receptor inhibitors - is there more to come? Eur J Cancer 2013; 49: 2504-2511

44 Choueiri TK, Pal SK, McDermott DF et al. A phase I study of cabozantinib (XL184) in patients with renal cell cancer. Ann Oncol 2014; 25 : 1603-1608

45 Topalian SL, Hodi FS, Brahmer JR et al. Safety, activity, and immune correlates of anti-PD-1 antibody in cancer. N Engl J Med 2012; 366: 2443-2454

46 McDermott DF, Drake CG, Sznol M et al. Survival, Durable Response, and Long-Term Safety in Patients With Previously Treated Advanced Renal Cell Carcinoma Receiving Nivolumab. J Clin Oncol 2015

47 Rini BI, Halabi S, Rosenberg JE et al. Bevacizumab plus interferon alfa compared with interferon alfa monotherapy in patients with metastatic renal cell carcinoma: CALGB 90206. J Clin Oncol 2008; 26: 5422-5428

48 Sternberg CN, Hawkins RE, Wagstaff J et al. A randomised, doubleblind phase III study of pazopanib in patients with advanced and/ or metastatic renal cell carcinoma: final overall survival results and safety update. Eur J Cancer 2013; 49: 1287-1296

49 Motzer RJ, Escudier B, Tomczak $P$ et al. Axitinib versus sorafenib as second-line treatment for advanced renal cell carcinoma: overall survival analysis and updated results from a randomised phase 3 trial. Lancet Oncol 2013; 14: 552-562

50 Escudier B, Porta C, Schmidinger $M$ et al. Renal cell carcinoma: ESMO Clinical Practice Guidelines for diagnosis, treatment and follow-up. Ann Oncol 2014; 25: (Suppl 3): iii49-iii56 